Check for updates

Cite this: Nanoscale Adv., 2019, 1, 2337

\title{
Stretchable elastomer composites with segregated filler networks: effect of carbon nanofiller dimensionality $\dagger$
}

\begin{abstract}
Kai Ke, (D) * Zhen Sang and Ica Manas-Zloczower*
Electrically conductive elastomer composites (CECS) have great potential in wearable and stretchable electronic applications. However, it is often challenging to trade off electrical conductivity and mechanical flexibility in melt-processed CECs for wearable electronic applications. Here, we develop CECs with high electrical conductivity and mechanical elasticity by controlling the segregated networks of carbon nanofillers formed at the elastomer interface. The carbon nanofiller dimensionality has a significant influence on the electrical and mechanical properties of thermoplastic polyurethane (TPU) composites. For instance, 3D branched carbon nanotubes (carbon nanostructures, CNSs) have a very low percolation threshold $\left(\Phi_{C}=0.01\right.$ wt\%), which is about 8-10 times lower than that of $1 \mathrm{D}$ carbon nanotubes (CNTs) and 2D graphene nanosheets (GNSs). Besides, the TPU/CNS system has a higher electrical conductivity than other fillers at all filler contents (0.05-2 wt\%). On the other hand, TPU/CNT systems can retain high elongation at break, whereas for the TPU/GNS systems elongation at break is severely deteriorated, especially at a high filler content. Different electrical and mechanical properties in the TPU-based CECs enable potential applications in flexible conductors/resistors and stretchable strain sensors, respectively.
\end{abstract}

Received 21st March 2019

Accepted 24th April 2019

DOI: 10.1039/c9na00176j

rsc.li/nanoscale-advances

\section{Introduction}

The rise of the Internet of Things makes the development of flexible electronic materials of great demand for humanmachine interface bridging. As the human skin is compliant to any curved structure, lightweight conductive elastomer composites (CECs) with excellent flexibility and stretchability are very good alternatives to conventional metal-based conductive materials for wearable electronics. Currently, CECs have been widely used in stretchable conductors, ${ }^{1-8}$ wearable strain sensors, ${ }^{9-19}$ and flexible pressure sensors. ${ }^{20-27}$

As elastomers are intrinsically insulating, it is essential to incorporate conductive materials into elastomers for the fabrication of CECs. There are three main reported strategies: (1) infiltrating elastomers with conductive filler networks, ${ }^{9,10,28,29}$ (2) synthesizing metal fillers within elastomers $^{3,28,30-32}$ and (3) implanting conductive fillers into elastomers. ${ }^{12,21,22,26,27,33-45}$ The first strategy generally requires particular fabrication techniques, such as surface treatment, to assist the combination of elastomers with conductive fillers. Besides, conductive filler networks built on the surface

Department of Macromolecular Science and Engineering, Case Western Reserve University, 2100 Adelbert Road, Cleveland, OH 44106-7202, USA. E-mail: kxk424@ case.edu;ixm@case.edu

† Electronic supplementary information (ESI) available. See DOI: 10.1039/c9na00176j or in the subsurface of the elastomers may be damaged under long-term high-level strain service duration due to the weak bonding between filler networks and elastomers. Synthesizing metal fillers within elastomers is an effective method to combine elastomers and conductive fillers by forming uniform filler networks in elastomers. However, it is only suitable for incorporating metal nanoparticles into elastomers, and the elastomer solution or latex is a prerequisite for the dispersion and reduction reaction of metal precursors. As such, implanting conductive fillers into elastomers by either solution or melt mixing, is relatively low-cost and facile, and thus widely reported in the literature. Nevertheless, the electrical conductivity depends on the percolation behavior of conductive fillers dispersed in the elastomers and the composites become electrically conductive only if the filler content reaches a critical content, i.e. percolation threshold $\left(\Phi_{\mathrm{C}}\right)$. In comparison with solution and melt mixing, the formation of segregated filler networks at the polymer interface is a more efficient strategy to reduce $\Phi_{\mathrm{C}}$ and to achieve good electrical conductivity for polymer composites. ${ }^{38,46-54}$ In this strategy, conductive fillers are trapped at the interface of polymer particles in contact with each other, leading to economic and efficient formation of conductive paths along the polymer-polymer interface. However, due to the weak polymer-filler interfacial interactions and bad filler distribution at the interface, the resultant composites usually have deteriorated elongation at break when compared with those 
fabricated via solution/melt mixing, especially at high filler loading. ${ }^{51,54-56}$ As such, these composites have high potential for electromagnetic interference shielding ${ }^{52,54}$ and thermoelectric applications, ${ }^{51,57-59}$ which focus more on electrical conductivity. However, the fabrication of stretchable electronics often requires both high electrical conductivity and high stretchability. It is challenging to realize this in segregated CECs by using common carbon nanofillers at low filler loading.

Recently, graphene foam (three-dimensional, 3D) has become an attractive alternative to common carbon nanofillers for the fabrication of conductive polymer composites due to their excellent conductivity. ${ }^{\mathbf{1 6 , 6 0 - 6 4}}$ Likewise, branched carbon nanotubes (CNSs) (3D) are superior to multi-walled carbon nanotubes (CNTs) (1D) or graphene nanoplatelets (2D) for improving the electrical conductivity of melt-mixed polymer composites, $^{34,65}$ in alignment with theoretical studies. ${ }^{66}$ However, for segregated network structures, the literature suggests that carbon nanofillers with lower dimensionality tend to have lower $\Phi_{\mathrm{C}}$ values and higher electrical conductivity at the same filler loading in thermoplastic nanocomposites. ${ }^{49,67}$ The rationale for this observation is the agglomeration of $2 \mathrm{D}$ graphene caused by filler stacking as well as the plane-to-plane contact in comparison with the end-to-end contact for $1 \mathrm{D}$ CNTs. In addition, the resultant composites often have severely deteriorated elongation at break. Therefore, exploration of effective strategies to improve filler dispersion and to enhance the filler-polymer interface is crucial for simultaneously achieving high elongation at break and electrical conductivity in the CECs.

In our previous work, it was found that the formation of segregated CNS networks at the interface of thermoplastic polyurethane (TPU), which is a widely used elastomer for flexible sensors, ${ }^{\mathbf{1 7 , 2 5 , 3 9 , 4 2 , 4 4 , 6 8 , 6 9}}$ is able to significantly reduce $\Phi_{\mathrm{C}}$ of the TPU/CNS composites in comparison with melt mixing. ${ }^{70}$ Besides, the elastomer particle size plays an important role in controlling the filler network morphology in the segregated network structure, affecting the composite's electrical and mechanical properties. ${ }^{71}$ Another key factor affecting the polymer nanocomposite's electrical and mechanical properties significantly is nanofiller dimensionality. ${ }^{72}$ This factor has not been studied so far in CEC systems with segregated networks for piezoresistive sensing applications.

In this study we developed thermoplastic polyurethane composites with different carbon nanofillers (1D CNTs, 2D GNSs and 3D CNSs) using the brick-wall structure strategy, i.e. forming segregated filler networks at the elastomer interface. We aim to obtain elastomer composites with high electrical conductivity (>1 $\left.\mathrm{S} \mathrm{m}^{-1}\right)$ and large elongation at break $(>1000 \%)$ simultaneously to ensure stretchable and wearable electronic applications. The morphology, electrical and mechanical properties, as well as piezoresistive behaviors of the composites with the three types of carbon nanofillers were comparatively studied. Moreover, potential applications of these stretchable TPU-based CECs for piezoresistive strain sensors and resistors were demonstrated.

\section{Experimental}

\subsection{Materials and composite preparation}

Commercial TPU consisting of $p$-phenylenediisocyanates and polycaprolactone polyols was used here. Carbon nanostructures CNSs (known as branched carbon nanotubes; CNS flake length $70 \mu \mathrm{m}$ and thickness $10 \mu \mathrm{m}$; nanotube diameter $9 \mathrm{~nm}$ ) were provided by Applied Nanostructured Solutions LLC (Lockheed Martin Corporation, MD, US). Multi-walled carbon nanotubes (CNTs, nanocyl NC7000, diameter $9.5 \mathrm{~nm}$ and length $1 \mu \mathrm{m}$ ) and single layer graphene (GNSs, Angstron N002-PDR, lateral length $10 \mu \mathrm{m}$ and thickness $1 \mathrm{~nm}$ ) were also used.

TPU composites were prepared by grinding the TPU with an IKA grinder (MF 10) and sifting the particles to obtain a particle size of 212-300 $\mu \mathrm{m}$. Smaller particle sizes possess more interfacial area, ${ }^{53}$ which is beneficial for the interfacial bonding between fillers and TPU, enabling good mechanical properties. Carbon nanofillers were dispersed in ethanol ( $c a .40 \mathrm{ml}$ ) under sonication for $10 \mathrm{~min}(700 \mathrm{~W}, 45 \%$ amplitude, $5 \mathrm{~s}$ on and $5 \mathrm{~s}$ off) to achieve better dispersion (see Fig. S1 in the ESI $\dagger$ ). The TPU powder was added into the filler suspension and mixed for 20 min under magnetic stirring at room temperature. Ultrasonication improves filler dispersion conducive to property improvement in comparison with hand mixing reported in our previous work, ${ }^{70}$ as confirmed by the results in Fig. S2 in the ESI. $\uparrow$ The obtained mixture was dried in an oven for $36 \mathrm{~h}$ at $90{ }^{\circ} \mathrm{C}$ to remove residual ethanol and moisture prior to compression molding. Finally, the powdery mixture was compression molded into rectangular films $\left(11 \mathrm{~cm} \times 11 \mathrm{~cm}, 0.7 \mathrm{~mm}\right.$ thick) at $230{ }^{\circ} \mathrm{C}$ under $5 \mathrm{MPa}$ for $5 \mathrm{~min}$. The TPU nanocomposites were labeled according to their composition, e.g. the nanocomposites with $0.7 \mathrm{wt} \% \mathrm{CNSs}$ were labeled as $0.7 \mathrm{CNS}$.

\subsection{Characterization}

TEM images of the fillers were obtained using a FEI Tecnai F30 transmission electron microscope. The as-received fillers were dispersed in $\mathrm{N}, \mathrm{N}$-dimethylformamide using ultrasonication prior to TEM analysis.

Optical microscope images (Olympus BX51TF, Tokyo, Japan) were used to analyze the network structure of the three types of carbon nanofillers in the TPU matrix. Thin films $(3 \mu \mathrm{m}$ thick $)$ were cut using a microtome (LEICA EM FC6) at $-80{ }^{\circ} \mathrm{C}$.

A scanning electron microscope (SEM) Nova NanoLab 650 FEG-SEM/FIB (Hillsboro, OR) was used to observe the morphology of TPU nanocomposites. Prior to SEM observation, palladium was sputter coated on the cryo-fractured specimens.

The electrical resistance of TPU films was measured using a resistance system set (PRS-801, Prostat Corporation) as reported in the literature. ${ }^{34,73}$ Nine dumbbell-shaped specimens (30 $\mathrm{mm}$ length, $4.94 \mathrm{~mm}$ width and $0.75 \mathrm{~mm}$ thickness) cut from compression molded films were measured $(10 \mathrm{~mm}$ between clamp electrodes) to get an average.

A uniaxial tensile test was performed using an MTS instrument (MTS Systems, US, MN) with a loading speed of 50 $\mathrm{mm} \min ^{-1}$ at room temperature. Nine dog-bone-shaped samples were tested for each composition. 
The piezoresistive behavior was investigated by measuring the instantaneous resistance change using a digital multimeter (Keithley 2701, OH, US) with a loading speed of $5 \mathrm{~mm} \mathrm{~min}^{-1}$ (MTS system, MN, US) at room temperature. All tested samples were subjected to tensile break or resistance beyond $110 \mathrm{M} \Omega$ (the measure limit of the multimeter). Cyclic testing was performed with a tensile instrument (Zwick Roell Z0.5, Germany) at a maximum stress of $8.5 \mathrm{MPa}$ (corresponding to a maximum strain of $c a .45-60 \%$ ) and loading speed of $2 \mathrm{~mm} \mathrm{~min}^{-1}$. The holding time for the end of loading and unloading was 2 s. Strip strain sensors made of 0.3 CNS composite films $(0.7 \mathrm{~mm}$ thick, $2 \mathrm{~mm}$ wide, and $70 \mathrm{~mm}$ long) were applied to demonstrate the motion detection of the knee, wrist and index finger by recording the resistance change using the Keithley multimeter. Copper wires were connected to the film strip ends painted with silver paste to ensure good contact between electrodes and samples. For the demonstration of flexible conductor/resistor applications, same-sized film strips made from the TPU composites with $2 \mathrm{wt} \%$ carbon nanofillers were connected to a circuit with LED bulbs powered by a DC power supply (BK Precision 1667, CA, US). The bulb brightness of non-stretched composites and composites stretched to $100 \%$ strain was compared to simply show their potential flexible conductor/resistor applications.

\section{Results and discussion}

\subsection{Challenges and design strategy}

The literature results in Table 1 show the difficulty in balancing electrical conductivity and elongation at break for TPU-based CECs fabricated via conventional melt or solution processing methods when using either graphene or multi-walled carbon nanotubes. To achieve high electrical conductivity $\left(>1 \mathrm{~S} \mathrm{~m}^{-1}\right)$, large amounts of common carbon nanofillers are generally consumed and in turn this leads to deterioration in the strain at break. In previous work, we proposed a facile strategy to build conductive networks at the TPU-TPU interface, i.e. a segregated filler network structure, inspired by the brick wall structure. ${ }^{70}$ TPU composite films with segregated networks of carbon nanofillers were obtained by compression molding of filler coated TPU powders obtained by mixing TPU particles in a filler suspension and drying, as seen in Fig. 1. It is noteworthy that, during compression molding, the nanofillers with infiltrated TPU chains at the interface serve as the mortar, while the TPU granules are the bricks, as shown in the bottom left scheme in Fig. 1. In this case, the mortar serves as pathways for electron conduction, while also binding the TPU bricks. Although this strategy contains a few steps, it is environment-friendly and facile for the fabrication of CECs with both high electrical conductivity and high stretchability. However, the morphology and agglomeration of fillers at the elastomer interface affects the mortar layer properties (e.g. thickness and morphology), resulting in differences in electrical conductivity, stretchability and piezoresistive properties of the resultant TPU composites.

\subsection{Carbon nanofiller morphologies}

Fig. 2 shows TEM images of raw carbon materials, CNTs, GNSs and CNSs, dispersed in DMF. CNTs have a rod-like structure

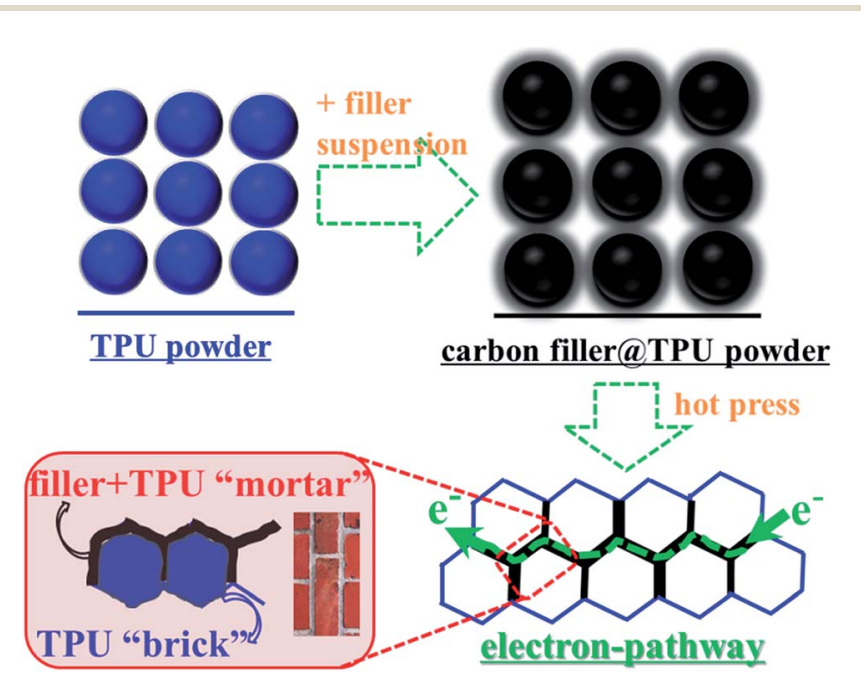

Fig. 1 Schematic illustrations of the fabrication of TPU composites with carbon nanofillers and the "brick-wall" structure in TPU composites.

Table 1 Stretchability and electrical conductivity of TPU-based CECs ${ }^{a}$

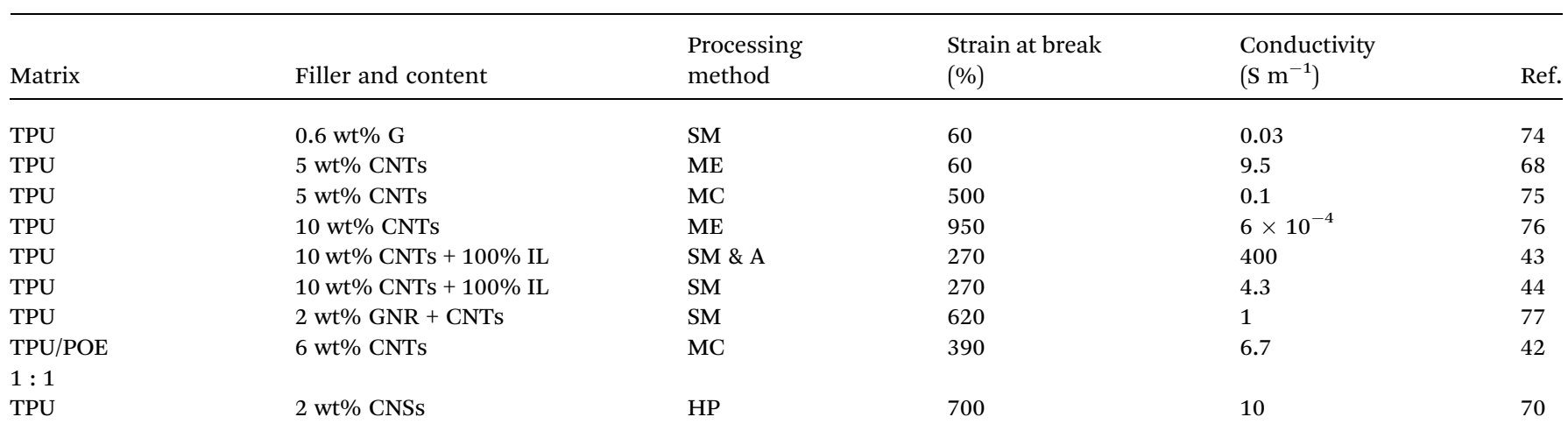

${ }^{a}$ G: graphene. IL: ionic liquid. GNR-CNTs: graphene nanoribbon and carbon nanotubes, prepared by unzipping MWCNTs. POE: polyolefin elastomer. SM: solution mixing. SM \& A: solution mixing and annealing. ME: melt extrusion. MC: melt compounding. HP: hot pressing. 

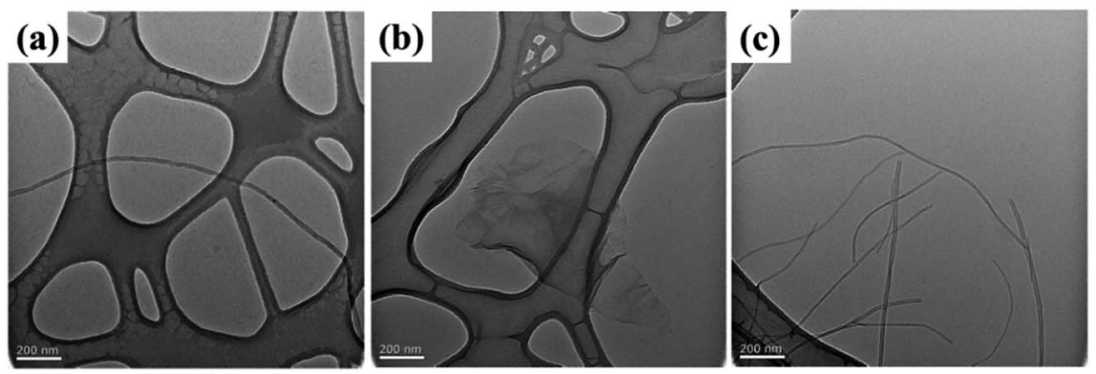

Fig. 2 TEM images of (a) CNTs, (b) GNSs and (c) CNSs.

(1D), and the TEM image reveals string-like single carbon nanotubes when dispersed in DMF under ultrasonication. GNSs exhibit a plate-like morphological structure (2D), containing 12 layers of wrinkled graphene, which is thin and transparent. CNSs display a branch-like morphology with obvious "arms" which tend to interconnect with each other to form a "crosslinked" structure (3D).

\subsection{Morphologies of carbon nanofillers at the elastomer interface}

To observe the segregated network structure of all three carbon nanofillers, optical microscopy images are shown in Fig. 3. With increasing filler content, the segregated network becomes clearer and more interconnected, especially at high concentration. However, in comparison with GNSs and CNSs, the segregated network in the TPU/CNT system is less obvious, which is probably due to the relatively low filler dimensionality conducive to filler bundling/entanglement. Interestingly, at even $0.1 \mathrm{wt} \%$, the segregated network for the TPU/CNS system is well marked, indicating the high efficiency in forming conductive pathways at the elastomer interface.
To further understand the differences in morphology of the three kinds of carbon nanofillers in the TPU matrix, SEM images of TPU composites with $0.7 \mathrm{wt} \%$ filler are shown in Fig. 4. Like in the optical microscopy images, the location of all carbon nanofillers at the elastomer interface is clearly observed. Besides, the corresponding magnified images on the right side indicate the penetration of TPU chains into the filler network. Dispersed single CNTs and bundles with a few nanotubes can be observed in the magnified SEM images on the right, while the GNSs display the typical morphology of wrinkled and stacked large graphene plates. In contrast, CNSs have a hair-like highly interconnected structure due to the branches or "arms" consistent with the TEM images in Fig. 2, and a tree root-soil structure is observed in the interfacial layer with TPU chains penetrated into the CNS network.

\subsection{Electrical conductivity}

The volume electrical conductivity of TPU composites with the three types of carbon nanofillers is presented in Fig. 5. The CNTs and GNSs selected here are frequently used commercial carbon nanofillers for electrically conductive polymer

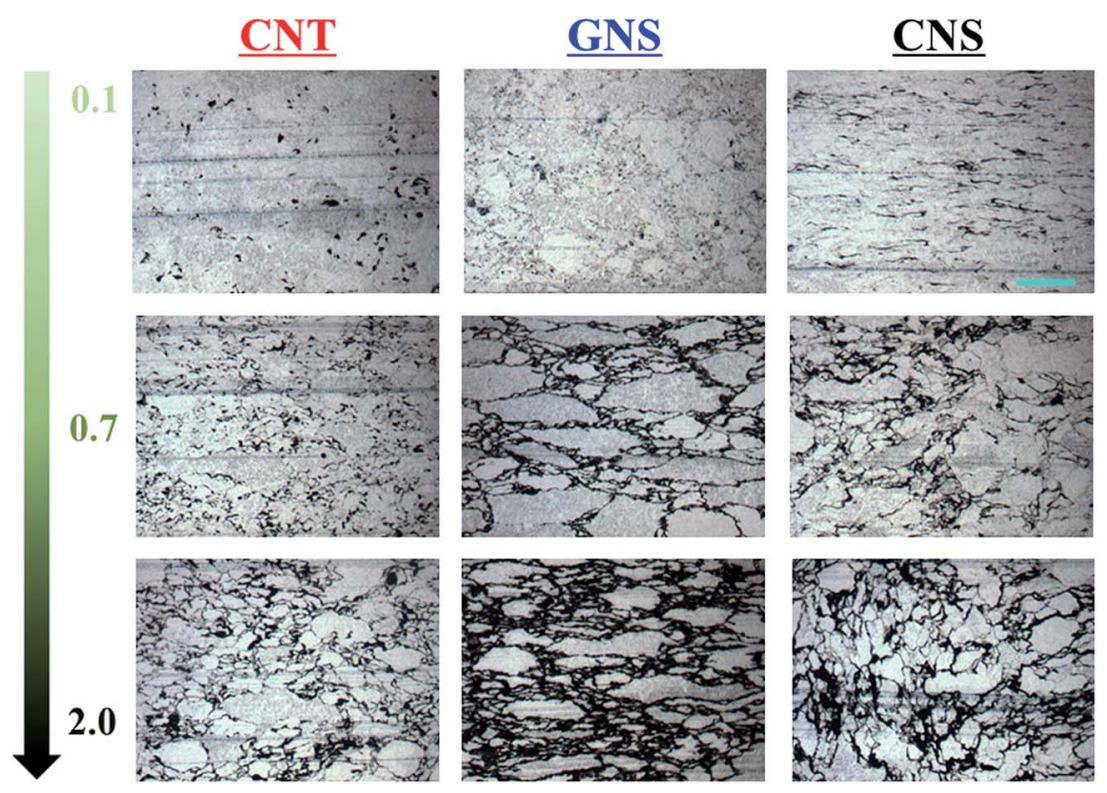

Fig. 3 Optical microscopy morphologies of CNTs, GNSs and CNSs at the TPU interface with filler contents of $0.1,0.7$ and 2 wt\%, respectively. The scale bar is $100 \mu \mathrm{m}$. 

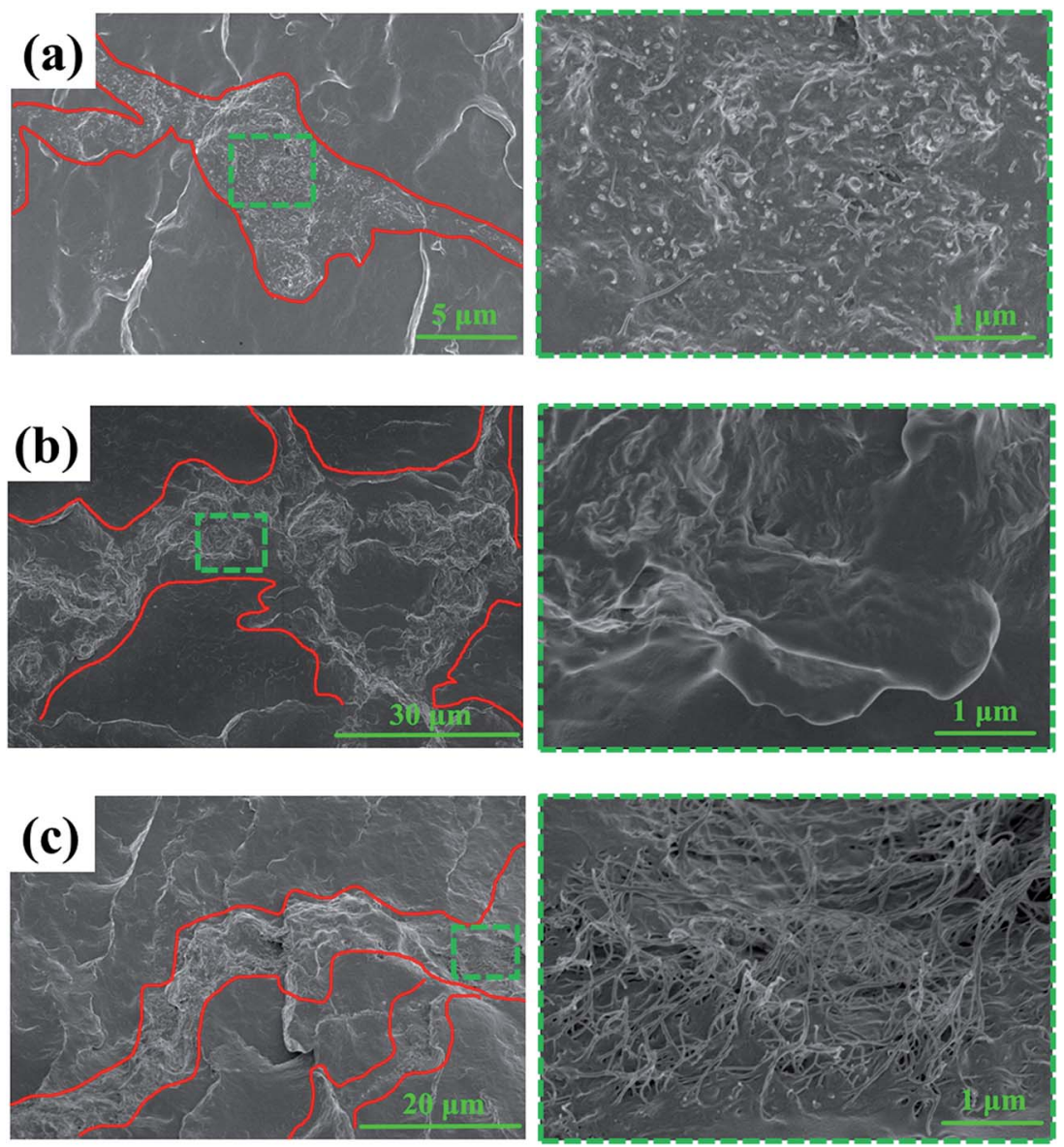

Fig. 4 SEM morphology of (a) CNTs, (b) GNSs and (c) CNSs at the TPU interface with a filler content of 0.7 wt\%.

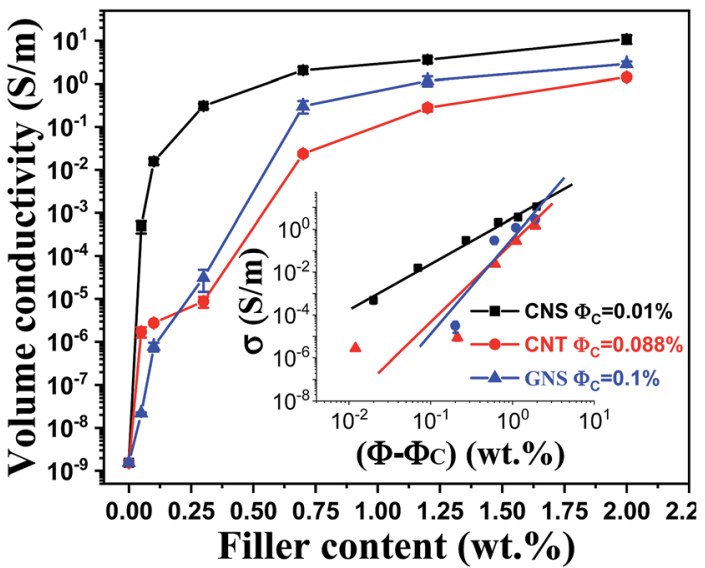

Fig. 5 Volume conductivity versus filler content for TPU composites.

composites. With the increasing filler content, the volume conductivity of TPU composites increases significantly after $0.3 \mathrm{wt} \%$ and it reaches a plateau once the filler content goes up to $1.2 \mathrm{wt} \%$. However, the incorporation of only $0.1 \mathrm{wt} \% \mathrm{CNSs}$ results in a dramatic improvement ( 5 orders of magnitude) of the volume conductivity for TPU composites, illustrating the much higher efficiency of CNSs for the formation of conductive paths at the TPU interface. Afterwards, the volume conductivity increases slowly (1-1.5 orders of magnitude) and it reaches almost a plateau at $0.7 \mathrm{wt} \%$ CNSs with much tapered increases at higher concentrations. Interestingly, at $0.3 \mathrm{wt} \%$ filler content, the TPU/CNS composite has a volume conductivity 4 orders of magnitude higher than that of the CNT and GNS composites. Modest conductivity increases for TPU composites with CNTs and GNSs at filler contents between 0.1 and $0.3 \mathrm{wt} \%$ are probably due to the filler stacking/agglomeration and/or inadequate connection at the elastomer interface (see Fig. 2 for the composites with $0.1 \%$ fillers). Moreover, the TPU/CNS system has higher electrical conductivity at all filler concentrations. The composite with $0.7 \mathrm{wt} \%$ CNSs has an electrical conductivity of $2.1 \mathrm{~S} \mathrm{~m}^{-1}$, which is even higher than that of $2 \mathrm{wt} \%$ CNTs (1.4 S $\mathrm{m}^{-1}$ ) and is similar to that of the TPU composite with $2 \mathrm{wt} \%$ GNSs $\left(2.9 \mathrm{~S} \mathrm{~m}^{-1}\right)$.

The classical percolation theory was used to calculate the percolation threshold for the different fillers: ${ }^{78,79}$

$$
\sigma \propto \sigma_{0}\left(\Phi-\Phi_{\mathrm{C}}\right)^{t}
$$

where $\sigma$ and $\sigma_{0}$ are the electrical conductivities of the composite and filler, respectively, $\Phi$ and $\Phi_{\mathrm{C}}$ represent the filler volume fraction and the percolation threshold and $t$ is a power-law constant related to the intrinsic filler conductivity. 
As shown in the inset in Fig. 5, the percolation threshold of TPU composites with CNTs or GNSs is 0.088 and $0.1 \mathrm{wt} \%$, respectively, while that for the TPU/CNS system is only $0.01 \mathrm{wt} \%$. This super low $\Phi_{\mathrm{C}}$ for the TPU/CNS system is attributed to the filler interconnected morphology, ${ }^{70,80}$ favorable for the formation of conductive paths at the TPU interface. This value is currently the lowest reported in the literature for TPU composites with carbon nanofillers, indicative of the higher efficiency of the segregated filler network strategy used in this work compared to solution or melt mixing. ${ }^{26,39,74}$

Overall, the 3D CNSs show higher efficiency in forming conductive pathways at the elastomer interface in comparison with 2D GNSs and 1D CNTs, which is consistent with theoretical studies $^{66}$ while different from other segregated network systems. ${ }^{\mathbf{4 9 , 6 7}}$ This is likely due to the improved filler dispersion at the elastomer interface (see Fig. $\mathrm{S} 2 \dagger$ ) and the penetration of TPU chains into the CNS network (see Fig. 4).

\subsection{Mechanical properties}

The morphology of carbon nanofillers influences not only the electrical conductivities but also the mechanical properties of TPU composites (Fig. 6). As shown in Fig. 6(a), the TPU/CNT system exhibits the largest elongation at break with a value still around $1300 \%$ for the composite with $2 \mathrm{wt} \%$ CNTs. In contrast, the incorporation of GNSs gives rise to a marked decrease in the elongation at break (the 0.7 and 2 wt $\%$ GNS composites have elongations at break of $900 \%$ and $500 \%$, respectively). This may be caused by the agglomeration of GNSs due to the stacking of graphene sheets, as shown in Fig. 4(b). The TPU/CNS composites also show lower elongation at break than the TPU/CNT system at all filler contents. The TPU/CNT system can effectively maintain a large elongation at break most likely due to the enhanced penetration of TPU chains into the looser CNT networks (see Fig. 3 and 4(a)) in comparison with the CNS and GNS systems. However, the elongations at break for TPU/CNS composites at very low filler loadings are still satisfactory $(1500 \%, 1400 \%$ and $1100 \%$ at loadings of $0.1,0.3$ and $0.7 \mathrm{wt} \%$, respectively), due to good filler dispersion at low loadings and the easy penetration of TPU chains into the CNS network. Such high elongations at break allow promising applications for stretchable conductive composites of these systems in view of their exceptional electrical conductivity. It is worth noting that these data are superior to those presented in Table 1 for the TPU composites fabricated by conventional methods. The relative Young's modulus data shown in Fig. 6(b) indicate that CNSs and GNSs have a comparable reinforcement effect, larger than that of the CNTs, most likely due to CNT bundling.

\subsection{Piezoresistive behaviors}

To explore the strain sensing applications of these stretchable composites, the relative resistance change $\left(\Delta R / R_{0}\right)$ plotted against strain is shown in Fig. 7. At $0.7 \mathrm{wt} \%$ filler content, the TPU/GNS system shows a much higher resistance change for 100-300\% strain in comparison with the other composites due most likely to the sliding between graphene sheets during stretching. ${ }^{26,80}$ It evolves quickly to complete separation of the graphene-graphene contact (at 300\% strain), and the resistance increases beyond the range of the multimeter $(110 \mathrm{M} \Omega)$, and yet this strain is lower than the composite elongation at break (850\%). This indicates that GNSs can potentially improve the strain sensitivity for TPU composites but not at high strains. Similar observations can be made for the $0.7 \mathrm{CNT}$, which can sustain relatively low tensile strains, e.g. $350 \%$, but resistance data cannot be collected beyond that due to the multimeter resistance limit. However, the TPU/CNS system can sustain strains up to $1100 \%$ with large $\Delta R / R_{0}$ values at a high strain level, most suitable for stretchable strain sensor applications. The robust conductive networks of CNSs at $0.7 \mathrm{wt} \%$ filler content enable the TPU/CNS system to sustain high levels of tensile strain. By further increasing the filler content to $2 \mathrm{wt} \%$ (Fig. 7(b)), the TPU/GNS system still shows much higher $\Delta R / R_{0}$ at relatively low strain (100-300\%) while the CNS system can sustain higher levels of strain (up to $650 \%$ when it breaks). Interestingly, at this filler content, the TPU/CNT system can be applied for a wider strain range (up to 950\%), similar to the composite with only 0.7 wt\% CNSs. Thus, carbon nanofiller dimensionality significantly affects the piezoresistive properties of the TPU composites. 2D GNSs shows high sensitivity at low strain levels due to easy sliding between the graphene sheets. 1D CNTs can sustain a high strain level even at high filler loading due to TPU chain penetration into a looser filler network.
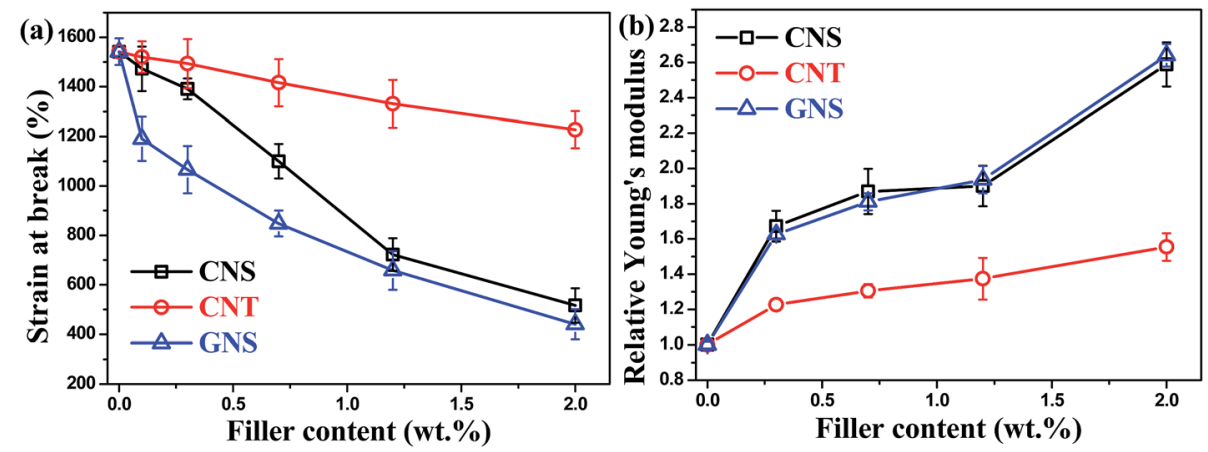

Fig. 6 (a) Elongation at break and (b) relative Young's modulus of TPU composites with different carbon nanofillers. 

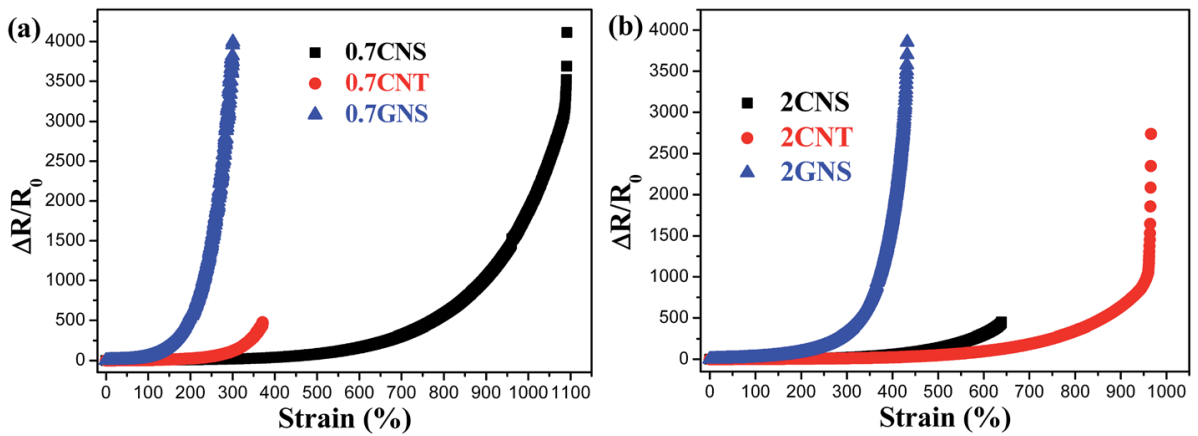

Fig. $7 \Delta R / R_{0}$ plotted against strain for TPU composites with (a) 0.7 and (b) 2 wt\% carbon nanofillers.

To further understand the effect of filler dimensionality on piezoresistive behavior, TPU composites with similar electrical conductivities but different contents of the three types of fillers are compared in Fig. 8(a and b). The 3D CNSs are superior to CNTs and GNSs for the fabrication of stretchable TPU composite strain sensors at low filler contents, while the
TPU/GNS system has higher piezoresistive sensitivity at strains of less than $300 \%$. To evaluate the strain sensitivity, gauge factors $\left(G_{\mathrm{F}}\right)$ calculated from the ratio of $\Delta R / R_{0}$ and strain change, i.e. relating the slopes of $\Delta R / R_{0}$-strain curves obtained by a linear fitting with the corresponding strain change, are provided in Fig. 8(a and b). As shown in Fig. 8(a), the TPU
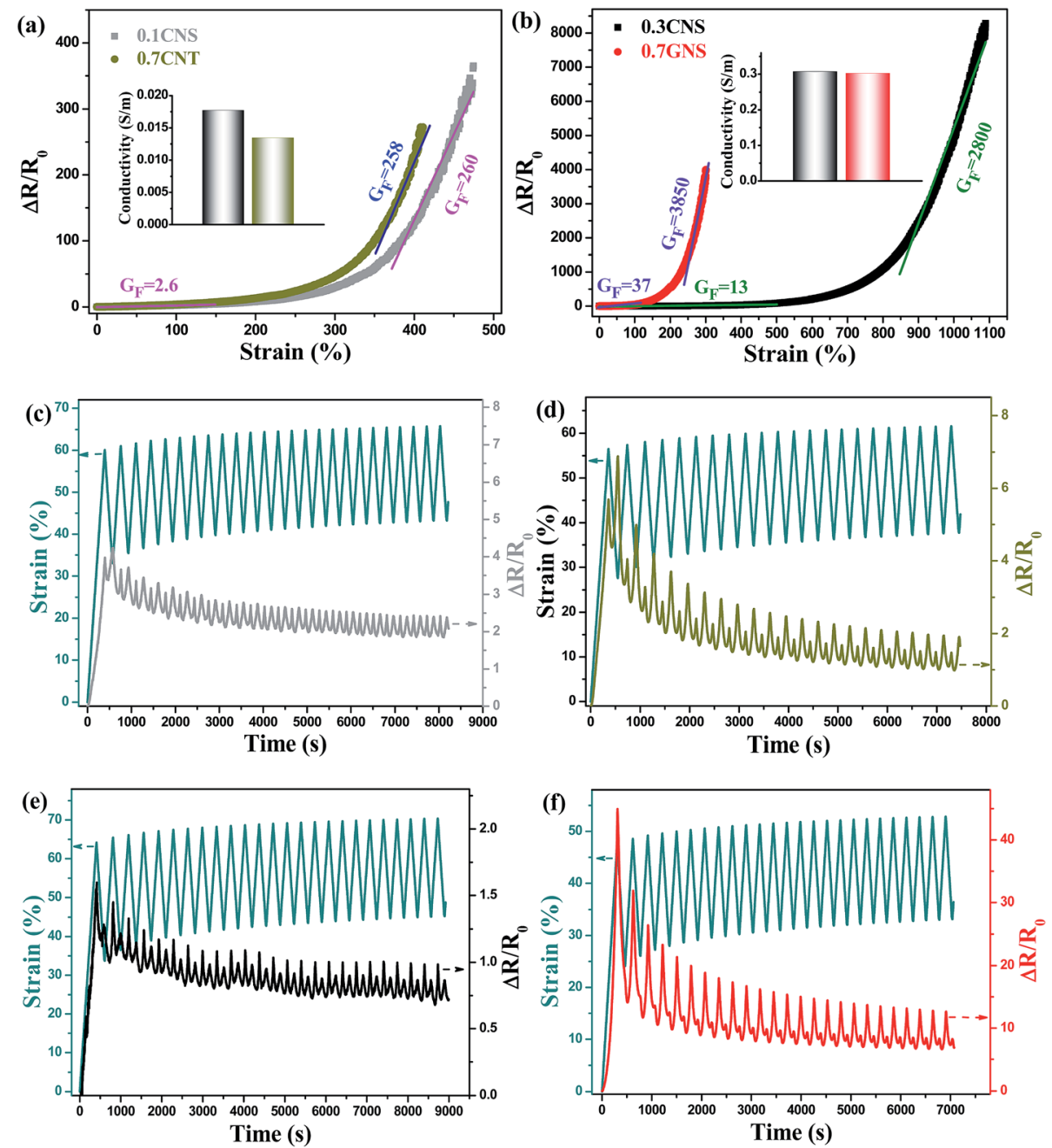

Fig. $8 \Delta R / R_{0}$-strain curves for the composites (a) $0.1 \mathrm{CNS}$ and $0.7 \mathrm{CNT}$ and (b) $0.3 \mathrm{CNS}$ and $0.7 \mathrm{GNS}$, as well as the $G_{\mathrm{F}}$ values for some strain regions. The inserts show their corresponding initial conductivities. Cyclic piezoresistive testing (under ca. $8.5 \mathrm{MPa}$ ) of TPU composites (c) 0.1 CNS, (d) 0.7 CNT, (e) 0.3 CNS and (f) 0.7 GNS, respectively. 
composites with $0.1 \mathrm{wt} \%$ CNSs and $0.7 \mathrm{wt} \%$ CNTs have very similar slopes for strain within $150 \%$ and strain above $350 \%$, indicating similar piezoresistive sensitivity. The gauge factor for TPU composites with both CNSs and CNTs within 150\% strain is about 2.6, comparable with that reported for solutionmixed TPU/CNT composites. ${ }^{44}$ Besides, they have higher $G_{\mathrm{F}}$ values (ca. 260) for strains above $350 \%$ by comparison with other literature reported stretchable strain sensors. ${ }^{\mathbf{8 1 , 8 2}}$ However, the composite with $0.7 \mathrm{wt} \%$ GNSs has a larger $G_{\mathrm{F}}(37)$ for low-level strain $(<100 \%)$ and, interestingly, $G_{\mathrm{F}}$ goes up to 3850 for strains between $250 \%$ and $300 \%$, which is much higher than that of other TPU-based stretchable strain sensors reported in the literature. ${ }^{\mathbf{8 1}}$ Although the TPU composite with $0.3 \mathrm{wt} \%$ CNSs has a lower $G_{\mathrm{F}}$ at strains within $400 \%$, it is able to sustain high-level strains and shows a $G_{\mathrm{F}}$ of 2800 for strains of $850-1050 \%$, much higher than that for other reported highly stretchable strain sensors. ${ }^{\mathbf{8 1 , 8 3}}$ The stability of these composites was tested by cyclic testing as illustrated in Fig. 8(c-f). The cyclic testing results indicate that the TPU/CNS systems with 0.1 and $0.3 \mathrm{wt} \%$ filler show rather stable sensing behavior in spite of their relatively smaller $\Delta R / R_{0}$ amplitude in comparison with the $0.7 \mathrm{wt} \%$ CNTs and $0.7 \mathrm{wt} \%$ GNS composites. It is worth mentioning that, due to the competition between network de-construction and re-construction during the unloading and the creep characteristics of elastomer composites, the data show shoulder peaks, as also reported in other TPU composites. ${ }^{\mathbf{2 6 , 3 9 , 4 4 , 4 5 , 7 4 , 8 0}}$ Interestingly, these shoulder peaks become weaker for the composites with more robust conductive networks, as revealed in Fig. 8(e and f), indicating that such networks exhibit better recovery upon unloading: ${ }^{\mathbf{8 0}}$

The effect of carbon nanofiller dimensionality on the properties of TPU composites can be summarized as follows: 3D
CNSs provide very high electrical conductivity, relatively high elongation at break and good piezoresistive sensitivity; 2D GNSs enable high piezoresistive sensitivity, medium electrical conductivity, but severely reduced elongation at break; 1D CNTs provide the highest elongation at break but relatively low electrical conductivity. Consequently, judicious carbon nanofiller selection plays a crucial role in designing conductive elastomer composites for different applications.

\subsection{Applications of stretchable conductive composites}

3.7.1 Strain sensors. To demonstrate the potential use of these composites for strain sensor applications, strip strain sensors made using the 0.3 CNS composite were used as an example to detect body joint motion, as presented in Fig. 9. The results in Fig. 9(a) reveal that the sensor displays corresponding piezoresistive responses to different knee bending angles, namely the larger the bending, the larger the $\Delta R / R_{0}$ signals. Moreover, it can also clearly detect wrist and finger bending (Fig. 9(b and c)), suggesting potential applications for body motion detection in healthcare..$^{\mathbf{9 1 1}, 33}$

3.7.2 Flexible conductors/resistors. Besides potential application for strain sensors, TPU composites with high carbon nanofiller contents can also be used as stretchable conductors. Fig. 10 illustrates a minimal change in resistance for the composites with $2 \mathrm{wt} \%$ CNTs or CNSs when stretched at $100 \%$ strain, whereas the composite with 2 wt $\%$ GNSs shows an eighteen fold increase in resistance at the same strain. Correspondingly, there is no change in the LED bulb brightness when the circuit is connected to the DC power supply using composite strips 2 CNS or 2 CNT, but there is a slight change in the brightness when connected with the composite resistor made using the 2 GNS strip. Thus TPU composites with a high content

(a)
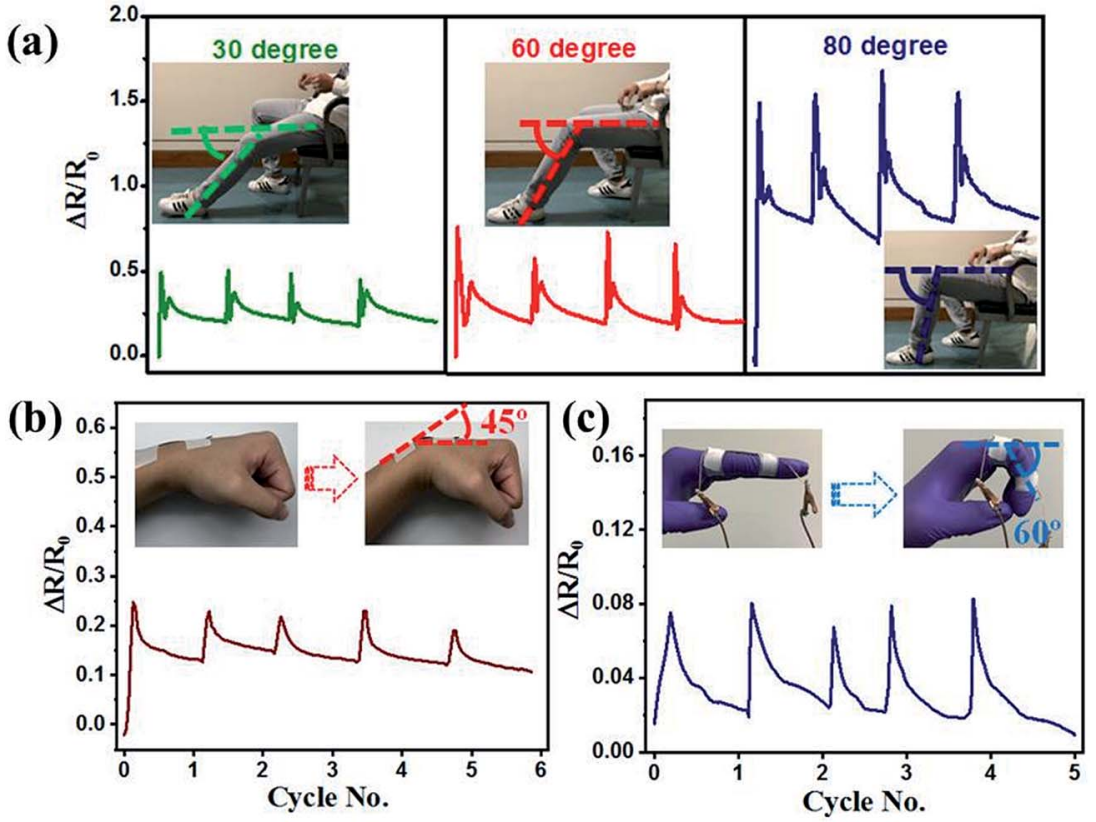

Fig. 9 Strain sensor applications for joint motion detection: (a) knee bending and (b) wrist and (c) index finger bending. 

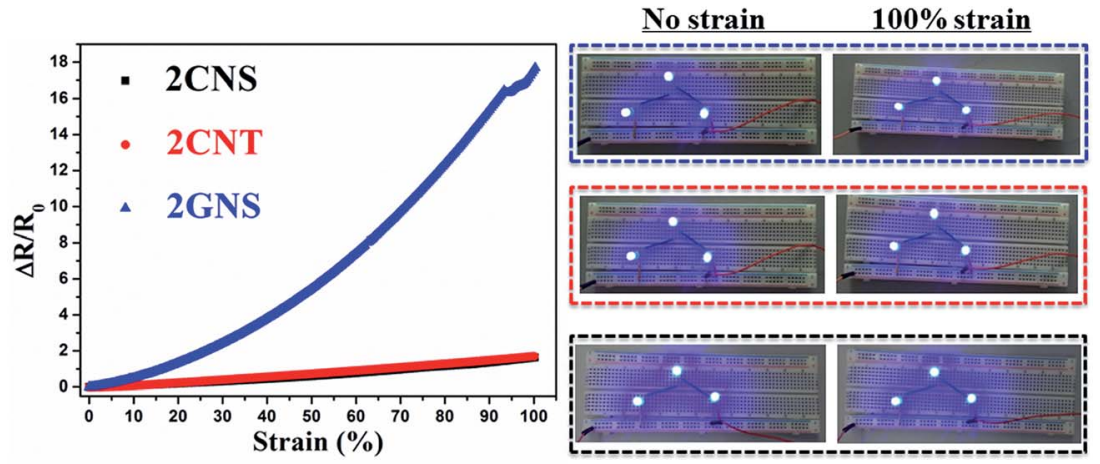

Fig. $10 \Delta R / R_{0}$ plotted against strain for $0-100 \%$ strain and the LED bulb brightness change under 0 and $100 \%$ strain, respectively, for TPU composites with different carbon nanofillers.

of CNSs or CNTs can be potentially used for stretchable conductors/resistors.

\section{Conclusion}

Electrically conductive thermoplastic elastomer composites with 1D CNTs, 2D GNSs and 3D CNSs were fabricated by building conductive pathways at the elastomer interface via a segregated network strategy. Comparing the three fillers, 3D CNSs have the lowest electrical percolation threshold (0.01 wt\%) and show much higher electrical conductivity than CNTs or GNSs at the same filler loading, especially at low filler content. In terms of composite flexibility, the TPU/CNT and TPU/GNS systems have the highest and lowest elongation at break, respectively, due to different filler network morphologies as related to their different shape/dimensionality. The lower elongation at break in the TPU/GNS system translates into a higher piezoresistivity but a narrow sensing strain range. The TPU/CNS system shows a good combination of high electrical conductivity and high elongation at break. Good electrical conductivity and high elongation at break enable the potential application of these composites in stretchable strain sensors and conductors/resistors, as demonstrated by the examples given for human body motion detection and flexible wires. This study sheds light on the effect of filler shape/dimensionality on the electrical, mechanical and piezoresistive properties of TPU composites with a segregated network structure and opens the way for their use as single or hybrid fillers in different applications.

\section{Conflicts of interest}

There are no conflicts to declare.

\section{Acknowledgements}

We appreciate Parker Hannifin Corporation for the financial support of this work. We also thank Mr Dale Ashby and Mr Glenn Craig for useful discussion and suggestions. We greatly appreciate Applied Nanostructured Solutions LLC for providing CNSs for our research. The authors also thank Mrs Nanthawan
Avishai and Dr Danqi Wang (both from the Swagelok Center for Surface Analysis of Materials of Case Western Reserve University) for SEM and TEM analysis.

\section{References}

1 S. P. Lacour, S. Wagner, Z. Huang and Z. Suo, Appl. Phys. Lett., 2003, 82, 2404-2406.

2 T. Sekitani, H. Nakajima, H. Maeda, T. Fukushima, T. Aida, K. Hata and T. Someya, Nat. Mater., 2009, 8, 494.

3 M. Park, J. Im, M. Shin, Y. Min, J. Park, H. Cho, S. Park, M.-B. Shim, S. Jeon and D.-Y. Chung, Nat. Nanotechnol., 2012, 7, 803.

4 S. Yao and Y. Zhu, Adv. Mater., 2015, 27, 1480-1511.

5 J. A. Rogers, T. Someya and Y. Huang, science, 2010, 327, 1603-1607.

6 U.-H. Shin, D.-W. Jeong, S.-M. Park, S.-H. Kim, H. W. Lee and J.-M. Kim, Carbon, 2014, 80, 396-404.

7 Z. Bao and X. Chen, Adv. Mater., 2016, 28, 4177-4179.

8 Y. Liu, K. He, G. Chen, W. R. Leow and X. Chen, Chem. Rev., 2017, 117, 12893-12941.

9 E. Roh, B.-U. Hwang, D. Kim, B.-Y. Kim and N.-E. Lee, $A C S$ Nano, 2015, 9, 6252-6261.

10 M. Amjadi, A. Pichitpajongkit, S. Lee, S. Ryu and I. Park, ACS Nano, 2014, 8, 5154-5163.

11 T. Yamada, Y. Hayamizu, Y. Yamamoto, Y. Yomogida, A. Izadi-Najafabadi, D. N. Futaba and K. Hata, Nat. Nanotechnol., 2011, 6, 296.

12 J.-H. Pu, X.-J. Zha, M. Zhao, S. Li, R.-Y. Bao, Z.-Y. Liu, B.-H. Xie, M.-B. Yang, Z. Guo and W. Yang, Nanoscale, 2018, 10, 2191-2198.

13 J. T. Muth, D. M. Vogt, R. L. Truby, Y. Mengüç, D. B. Kolesky, R. J. Wood and J. A. Lewis, Adv. Mater., 2014, 26, 6307-6312.

14 D. J. Lipomi, M. Vosgueritchian, B. C. Tee, S. L. Hellstrom, J. A. Lee, C. H. Fox and Z. Bao, Nat. Nanotechnol., 2011, 6, 788.

15 M. Amjadi, K. U. Kyung, I. Park and M. Sitti, Adv. Funct. Mater., 2016, 26, 1678-1698.

16 C. Yan, J. Wang, W. Kang, M. Cui, X. Wang, C. Y. Foo, K. J. Chee and P. S. Lee, Adv. Mater., 2014, 26, 2022-2027.

17 X. Li, T. Hua and B. Xu, Carbon, 2017, 118, 686-698. 
18 Y. Wang, J. Hao, Z. Huang, G. Zheng, K. Dai, C. Liu and C. Shen, Carbon, 2018, 126, 360-371.

19 Y. Li, B. Zhou, G. Zheng, X. Liu, T. Li, C. Yan, C. Cheng, K. Dai, C. Liu and C. Shen, J. Mater. Chem. C, 2018, 6, 2258-2269.

20 C. L. Choong, M. B. Shim, B. S. Lee, S. Jeon, D. S. Ko, T. H. Kang, J. Bae, S. H. Lee, K. E. Byun and J. Im, Adv. Mater., 2014, 26, 3451-3458.

21 M. Wang, K. Zhang, X.-X. Dai, Y. Li, J. Guo, H. Liu, G.-H. Li, Y.-J. Tan, J.-B. Zeng and Z. Guo, Nanoscale, 2017, 9, 1101711026.

22 H. Zhao and J. Bai, ACS Appl. Mater. Interfaces, 2015, 7, 96529659.

23 J. Wang, J. Jiu, M. Nogi, T. Sugahara, S. Nagao, H. Koga, P. He and K. Suganuma, Nanoscale, 2015, 7, 2926-2932.

24 J. Park, Y. Lee, J. Hong, M. Ha, Y.-D. Jung, H. Lim, S. Y. Kim and H. Ko, ACS Nano, 2014, 8, 4689-4697.

25 W. Huang, K. Dai, Y. Zhai, H. Liu, P. Zhan, J. Gao, G. Zheng, C. Liu and C. Shen, ACS Appl. Mater. Interfaces, 2017, 9, 42266-42277.

26 H. Liu, J. Gao, W. Huang, K. Dai, G. Zheng, C. Liu, C. Shen, X. Yan, J. Guo and Z. Guo, Nanoscale, 2016, 8, 12977-12989.

27 M. Knite, V. Teteris, A. Kiploka and J. Kaupuzs, Sens. Actuators, A, 2004, 110, 142-149.

28 M. Park, J. Park and U. Jeong, Nano Today, 2014, 9, 244-260.

29 L. Ma, W. Yang, Y. Wang, H. Chen, Y. Xing and J. Wang, Compos. Sci. Technol., 2018, 165, 190-197.

30 Y. Wei, S. Chen, Y. Lin, Z. Yang and L. Liu, J. Mater. Chem. C, 2015, 3, 9594-9602.

31 S. Chen, Y. Wei, S. Wei, Y. Lin and L. Liu, ACS Appl. Mater. Interfaces, 2016, 8, 25563-25570.

32 J. R. Dunklin, G. T. Forcherio, K. R. Berry Jr and D. K. Roper, ACS Appl. Mater. Interfaces, 2013, 5, 8457-8466.

33 S. Zhao, J. Li, D. Cao, G. Zhang, J. Li, K. Li, Y. Yang, W. Wang, Y. Jin and R. Sun, ACS Appl. Mater. Interfaces, 2017, 9, 1214712164.

34 K. Ke, V. S. Bonab, D. Yuan and I. Manas-Zloczower, Carbon, 2018, 139, 52-58.

35 T. S. Natarajan, S. B. Eshwaran, K. W. Stöckelhuber, S. Wießner, P. Pötschke, G. Heinrich and A. Das, ACS Appl. Mater. Interfaces, 2017, 9, 4860-4872.

36 L.-F. Ma, R.-Y. Bao, R. Dou, Z.-Y. Liu, W. Yang, B.-H. Xie, M.-B. Yang and Q. Fu, J. Mater. Chem. A, 2014, 2, 1698916996.

37 T. Li, J.-H. Pu, L.-F. Ma, R.-Y. Bao, G.-Q. Qi, W. Yang, B.-H. Xie and M.-B. Yang, Polym. Chem., 2015, 6, 7160-7170.

38 T. Li, L.-F. Ma, R.-Y. Bao, G.-Q. Qi, W. Yang, B.-H. Xie and M.-B. Yang, J. Mater. Chem. A, 2015, 3, 5482-5490.

39 Y. Zheng, Y. Li, K. Dai, M. Liu, K. Zhou, G. Zheng, C. Liu and C. Shen, Composites, Part A, 2017, 101, 41-49.

40 R. Zhang, H. Deng, R. Valenca, J. Jin, Q. Fu, E. Bilotti and T. Peijs, Compos. Sci. Technol., 2013, 74, 1-5.

41 M. Ji, H. Deng, D. Yan, X. Li, L. Duan and Q. Fu, Compos. Sci. Technol., 2014, 92, 16-26.

42 H. Deng, M. Ji, D. Yan, S. Fu, L. Duan, M. Zhang and Q. Fu, J. Mater. Chem. A, 2014, 2, 10048-10058.
43 L. Lin, S. Liu, S. Fu, S. Zhang, H. Deng and Q. Fu, Small, 2013, 9, 3620-3629.

44 L. Lin, S. Liu, Q. Zhang, X. Li, M. Ji, H. Deng and Q. Fu, ACS Appl. Mater. Interfaces, 2013, 5, 5815-5824.

45 S. Xu, W. Yu, M. Jing, R. Huang, Q. Zhang and Q. Fu, J. Phys. Chem. C, 2017, 121, 2108-2117.

46 J. C. Grunlan, W. W. Gerberich and L. F. Francis, J. Appl. Polym. Sci., 2001, 80, 692-705.

47 L. F. Francis, J. C. Grunlan, J. Sun and W. Gerberich, Colloids Surf., A, 2007, 311, 48-54.

48 X.-Y. Qi, D. Yan, Z. Jiang, Y.-K. Cao, Z.-Z. Yu, F. Yavari and N. Koratkar, ACS Appl. Mater. Interfaces, 2011, 3, 3130-3133.

49 J. Du, L. Zhao, Y. Zeng, L. Zhang, F. Li, P. Liu and C. Liu, Carbon, 2011, 49, 1094-1100.

50 J.-F. Gao, Z.-M. Li, Q.-j. Meng and Q. Yang, Mater. Lett., 2008, 62, 3530-3532.

51 H. Pang, L. Xu, D.-X. Yan and Z.-M. Li, Prog. Polym. Sci., 2014, 39, 1908-1933.

52 G. A. Gelves, M. H. Al-Saleh and U. Sundararaj, J. Mater. Chem., 2011, 21, 829-836.

53 J. Chen, Y.-y. Shi, J.-h. Yang, N. Zhang, T. Huang, C. Chen, Y. Wang and Z.-w. Zhou, J. Mater. Chem., 2012, 22, 2239822404.

54 D. X. Yan, H. Pang, B. Li, R. Vajtai, L. Xu, P. G. Ren, J. H. Wang and Z. M. Li, Adv. Funct. Mater., 2015, 25, 559566.

55 J. C. Grunlan, W. W. Gerberich and L. F. Francis, Polym. Eng. Sci., 2001, 41, 1947-1962.

56 H. Pang, D.-X. Yan, Y. Bao, J.-B. Chen, C. Chen and Z.-M. Li, J. Mater. Chem., 2012, 22, 23568-23575.

57 C. Yu, Y. S. Kim, D. Kim and J. C. Grunlan, Nano Lett., 2008, 8, 4428-4432.

58 C. Yu, K. Choi, L. Yin and J. C. Grunlan, ACS Nano, 2011, 5, 7885-7892.

59 H. Pang, Y.-Y. Piao, Y.-Q. Tan, G.-Y. Jiang, J.-H. Wang and Z.-M. Li, Mater. Lett., 2013, 107, 150-153.

60 Y. A. Samad, Y. Li, A. Schiffer, S. M. Alhassan and K. Liao, Small, 2015, 11, 2380-2385.

61 J. Li, S. Zhao, X. Zeng, W. Huang, Z. Gong, G. Zhang, R. Sun and C.-P. Wong, ACS Appl. Mater. Interfaces, 2016, 8, 1895418961.

62 J. Kuang, L. Liu, Y. Gao, D. Zhou, Z. Chen, B. Han and Z. Zhang, Nanoscale, 2013, 5, 12171-12177.

63 Y. Wu, Z. Wang, X. Liu, X. Shen, Q. Zheng, Q. Xue and J.-K. Kim, ACS Appl. Mater. Interfaces, 2017, 9, 9059-9069.

64 X. Liu, C. Tang, X. Du, S. Xiong, S. Xi, Y. Liu, X. Shen, Q. Zheng, Z. Wang and Y. Wu, Mater. Horiz., 2017, 4, 477-486.

65 B. Krause, C. Barbier, K. Kunz and P. Pötschke, Polymer, 2018, 159, 75-85.

66 S. H. Xie, Y. Y. Liu and J. Y. Li, Appl. Phys. Lett., 2008, 92, 243121.

67 P. G. Ren, Y. Y. Di, Q. Zhang, L. Li, H. Pang and Z. M. Li, Macromol. Mater. Eng., 2012, 297, 437-443.

68 J. F. Christ, N. Aliheidari, A. Ameli and P. Pötschke, Mater. Des., 2017, 131, 394-401.

69 H. Liu, Q. Li, S. Zhang, R. Yin, X. Liu, Y. He, K. Dai, C. Shan, J. Guo and C. Liu, J. Mater. Chem. C, 2018, 6, 12121-12141. 
70 Z. Sang, K. Ke and I. Manas-Zloczower, ACS Appl. Mater. Interfaces, 2018, 10(42), 36483-36492.

71 Z. Sang, K. Ke and I. Manas-Zloczower, ACS Appl. Polym. Mater., 2019, 1(4), 714-721.

72 R. Nadiv, G. Shachar, S. Peretz-Damari, M. Varenik, I. Levy, M. Buzaglo, E. Ruse and O. Regev, Carbon, 2018, 126, 410418.

73 H. Kim, Y. Miura and C. W. Macosko, Chem. Mater., 2010, 22, 3441-3450.

74 H. Liu, Y. Li, K. Dai, G. Zheng, C. Liu, C. Shen, X. Yan, J. Guo and Z. Guo, J. Mater. Chem. C, 2016, 4, 157-166.

75 J. Chen, Z.-x. Zhang, W.-b. Huang, J.-h. Yang, Y. Wang, Z.-w. Zhou and J.-h. Zhang, Mater. Des., 2015, 69, 105-113.

76 P. Costa, C. Silvia, J. Viana and S. L. Mendez, Composites, Part $B, 2014,57,242-249$.
77 M. Liu, C. Zhang, W. W. Tjiu, Z. Yang, W. Wang and T. Liu, Polymer, 2013, 54, 3124-3130.

78 L.-F. Ma, R.-Y. Bao, R. Dou, S.-D. Zheng, Z.-Y. Liu, R.-Y. Zhang, M.-B. Yang and W. Yang, Compos. Sci. Technol., 2016, 128, 176-184.

79 B. e. Kilbride, J. Coleman, J. Fraysse, P. Fournet, M. Cadek, A. Drury, S. Hutzler, S. Roth and W. Blau, J. Appl. Phys., 2002, 92, 4024-4030.

80 S. L. Yu, X. P. Wang, H. X. Xiang, L. P. Zhu, M. Tebyetekerwa and M. F. Zhu, Carbon, 2018, 140, 1-9.

81 Q. Fan, Z. Qin, S. Gao, Y. Wu, J. Pionteck, E. Mäder and M. Zhu, Carbon, 2012, 50, 4085-4092.

82 P. Slobodian, P. Riha and P. Sáha, Carbon, 2012, 50, 34463453.

83 G. Cai, J. Wang, K. Qian, J. Chen, S. Li and P. S. Lee, Adv. Sci., 2016, 4, 1600190-1600196. 